Ann. Génét. Sél. anim., I971, 3 (2), I53-160.

\title{
CORRÉLATIONS ENTRE LES PERFORMANCES D'ENGRAISSEMENT ET DE CARCASSE ET LES PERFORMANCES D'ÉLEVAGE CHEZ LE PORC
}

\author{
C. LEGAULT \\ avec la collaboration technique de Nathalie Boutser \\ Station de Génétique quantitative et appliquée, \\ Centre national de Recherches zootechniques, I.N.R.A., \\ 78 - Jouy-en-Josas
}

\begin{abstract}
RÉSUMÉ
Les corrélations entre les performances d'élevage (taille de la portée à la naissance et au sevrage, poids de la portée à 60 jours) de 1140 truies de race Large White et les performances d'engraissement (gain moyen quotidien de 30 à 100 kilogrammes et indice de transformation) et de carcasse (longueur, épaisseur du lard dorsal, \% de morceaux nobles et \% de morceaux gras) de 3 de leurs descendants issus de la même portée et contrôlés dans les stations de contrôle de la descendance ont été estimées. Les corrélations phénotypiques intra-verrat et station sont très faibles dans l'ensemble et non significatives. Trois coefficients dépassent cependant légèrement le seuil de signification $(P<0,05)$ : entre la longueur de la carcasse et la taille de la portée à la naissance $(r=-0,09)$ et au sevrage $(r=-0,10)$ et entre le pourcentage de morceaux nobles et le poids de la portée à 60 jours $(r=0,11)$. Les corrélations génétiques accentuent ces tendances sans qu'il soit possible de leur attribuer une valeur définitive. Enfin, les premiers coefficients de corrélation canonique montrent que la reproduction est pratiquement indépendante de la croissance $\left(\rho^{2}=0,002\right)$ et des caractéristiques de la carcasse $\left(\rho^{2}=0,028\right)$.

En conclusion, la sélection intensive, dont font actuellement l'objet les performances d'engraissement et de carcasse, ne devrait pas, du moins à court terme, être préjudiciable à la valeur reproductive des truies.
\end{abstract}

\section{I. - INTRODUCTION}

La sélection intensive dont font l'objet les performances d'engraissement et de carcasse aura-t-elle des répercussions sur les performances de reproduction de la truie? La recherche des corrélations génétiques et la vérification de la continuité des liaisons qu'elles représentent à l'occasion d'expériences de sélection sont les deux étapes logiques d'une réponse à cette question. L'existence de corré- 
lations génétiques élevées permet de prévoir le sens des effets indirects de la sélection. En revanche, comme le soulignaient $C_{\text {LAYTON }}$ et al. (I957), des corrélations génétiques faibles ne permettent pas, en raison des erreurs d'estimation, de prévoir dans quel sens vont évoluer à long terme les caractères liés.

En utilisant des informations recueillies dans les stations de contrôle de la descendance d'une part et dans les élevages d'autre part, nous nous proposons d'estimer ici les corrélations entre les caractères de production (liés à la croissance et à la conformation) et les caractères de reproduction chez la femelle.

\section{II. - MATÉRIEL ANIMAL ET MÉTHODES}

Deux ensembles de caractères $\mathrm{A}$ et $\mathrm{B}$ ont été pris en considération :

a) L'ensemble A comprend 6 caractères représentatifs des performances d'engraissement et de carcasse :

- le gain moyen quotidien de 30 à 100 kilogrammes, net,

- l'indice de transformation, exprimé en kilogramme d'aliment par kilogramme de poids

- le pourcentage de morceaux nobles (jambon et longe),

- le pourcentage de morceaux gras (bardière et panne),

- l'épaisseur du lard dorsal mesurée sur la carcasse,

- la longueur de la carcasse.

Pour chaque caractère, la variable étudiée est la moyenne des mesures relevées sur $n_{1}$ $=3$ porcs ( 2 femelles et 1 mâle castré) provenant de la même portée et contrôlés dans les stations de contrôle de la descendance de Jouy-en-Josas et de la Minière de 1963 à 1967.

b) L'ensemble $\mathrm{B}$ comprend 3 caractères représentatifs des performances d'élevage des truies dont l'une des portées a été contrôlée en station, comme nous venons de le décrire :

- le nombre de porcelets nés vivants par portée,

- le nombre de porcelets sevrés par portée,

- le poids de la portée à 60 jours.

Après correction pour l'effet du numéro de portée (LEgault, 1969), la valeur de la truie a été estimée pour chaque caractère en pondérant en fonction du nombre de ses portées connues conformément à la relation suivante (LusH, 1945):

dans laquelle :

$$
\mathrm{B}=\overline{\mathrm{X}}+\frac{n_{2} r}{1+\left(n_{2}-1\right) r}\left(\overline{\mathrm{X}}_{\mathrm{T}}-\overline{\mathrm{X}}\right)
$$

$\overline{\mathrm{X}}$ est la moyenne générale,

$\bar{X}_{\mathbf{T}}$ est la moyenne des $\mathbf{n}_{2}$ productions de la truie corrigées pour l'effet du numéro de portée,

$r \quad$ est la répétabilité du caractère considéré.

Les deux premières variables étaient connues sur l'ensemble des productions de 1140 truies de race Large White dont une portée avait été contrôlée en station alors que la troisième variable n'était disponible que pour 612 de ces animaux.

Les corrélations intra porcherie et verrat contrôlé $\left(R_{A B}\right)$ ont été estimées entre les variables $A$ et B; elles s'érrivent :

$$
\mathrm{R}_{\mathrm{AB}}=\frac{\text { Cov } \mathrm{AB}}{\sqrt{\mathrm{V}_{\mathrm{A}} \mathrm{V}_{\mathrm{B}}}}=\frac{\frac{1}{2} r_{\mathrm{Gab}} h_{a} h_{b \sigma_{a} \sigma_{b}}}{\sqrt{\mathrm{V}_{\mathrm{A}} \mathrm{V}_{\mathrm{B}}}}
$$

où $\sigma_{a}, \sigma_{b}, h_{a}$ et $h_{b}$ sont respectivement les écarts-types et les racines carrées des héritabilités des caractères reliés génétiquement par la corrélation $r_{\mathrm{Gab}}$. s'écrit :

$V_{A}$ est la variance de la moyenne d'une mesure prise sur $n_{1}$ individus d'une famille et

$$
\mathrm{V}_{\mathrm{A}}=\frac{1+\left(n_{1}-1\right) t}{n_{1}} \sigma_{a}^{2}
$$

où $t$ est le coefficient de corrélation phénotypique entre les performances des membres de cette famille.

$\mathrm{V}_{B}$ est la variance de la moyenne des $n_{2}$ performances d'une truie, et s'écrit:

où $r$ est la répétabilité du caractère.

$$
\mathrm{VB}=\frac{1+\left(n_{2}-1\right) r}{n_{2}} \sigma_{b}^{2}
$$


La corrélation génétique $r_{\mathrm{Gab}}$ s'écrit alors :

$$
r_{\mathrm{Gab}}=\frac{2 \mathrm{R}_{\mathrm{AB}}}{h_{\mathrm{a}} h_{\mathrm{b}}} \sqrt{\frac{\left[1+\left(n_{1}-1\right) t\right]\left[1+\left(n_{2}-1\right) r\right]}{n_{1} n_{2}}}
$$

Les valeurs des paramètres utilisés pour les calculs figurent au tableau 1.

TABLEAU I

Valeur des paramètres utilisés $\left(\mathrm{n}_{1}=\mathrm{n}_{2}=3\right.$ )

\begin{tabular}{|c|c|c|c|c|}
\hline $\begin{array}{c}\text { Origine } \\
\text { des données }\end{array}$ & Caractères & $h^{2}$ & $\mathrm{t}$ & $r$ \\
\hline \multirow{6}{*}{$\begin{array}{l}\text { OLLIVIER (I969) } \\
\text { Ensemble A }\end{array}$} & Gain moyen quotidien . . . . . & 0,447 & 0,4 I 6 & \\
\hline & Indice de transformation $\ldots . .$. & 0,473 & 0,392 & \\
\hline & $\%$ Jambon + longe....... & 0,537 & 0,299 & \\
\hline & $\%$ Bardière + panne . . . . . . . & 0,620 & $0,4 \times 8$ & \\
\hline & Épaisseur du lard dorsal . . . . . . & 0,723 & 0,433 & \\
\hline & Longueur de la carcasse...... . & 0,610 & 0,341 & \\
\hline \multirow{3}{*}{$\begin{array}{c}\text { LEGAULT (I970) } \\
\text { Ensemble } B\end{array}$} & Taille de la portée à la naissance. & 0,120 & & $0, \mathrm{~T} 50$ \\
\hline & Taille de la portée au sevrage . . & 0,100 & & 0,120 \\
\hline & Poids de la portée à 60 jours. . . & 0,080 & & 0,100 \\
\hline
\end{tabular}

L'ensemble de variables $A$ a été fractionné en deux sous-ensembles $A_{1}$ et $A_{2} ; A_{1}$ comprend les deux variables représentatives de la croissance (gain moyen quotidien et indice de transformation) alors que $\mathrm{A}_{2}$ comprend les quatre variables représentatives de la conformation et de la composition corporelle. Les premiers coefficients de corrélation canoniques entre $A_{1}$ et $B$ d'une part, et entre $\mathrm{A}_{2}$ et $\mathrm{B}$ d'autre part, ont alors été estimés selon la méthode décrite par Kendall (1957). Il s'agit des corrélations globales entre croissance et reproduction d'une part, et entre composition corporelle et reproduction d'autre part, ces trois ensembles étant représentés par les combinaisons linéaires des variables précitées qui maximisent ces corrélations.

\section{III. - RÉSULTATS}

Les corrélations phénotypiques entre variables liées à l'engraissement et à la composition corporelle d'une part, et les variables liées à la reproduction d'autre part figurent au tableau 2. Elles sont dans l'ensemble très faibles et non significatives. On observe cependant trois exceptions à cette règle pour des coefficients qui dépassent légèrement le seuil de signification; il existe d'une part une corrélation négative entre la longueur de la carcasse et la taille de la portée à la naissance ou au sevrage; d'autre part, on note l'existence d'une corrélation positive entre le pourcentage de morceaux nobles et le poids de la portée à 60 jours. 
Corrélations phénotypiques intra-porcherie et verrat entre les performances d'élevage de I I 40 truies de race Large white et la moyenne des performances d'engraissement et de carcasse de trois de leurs descendants

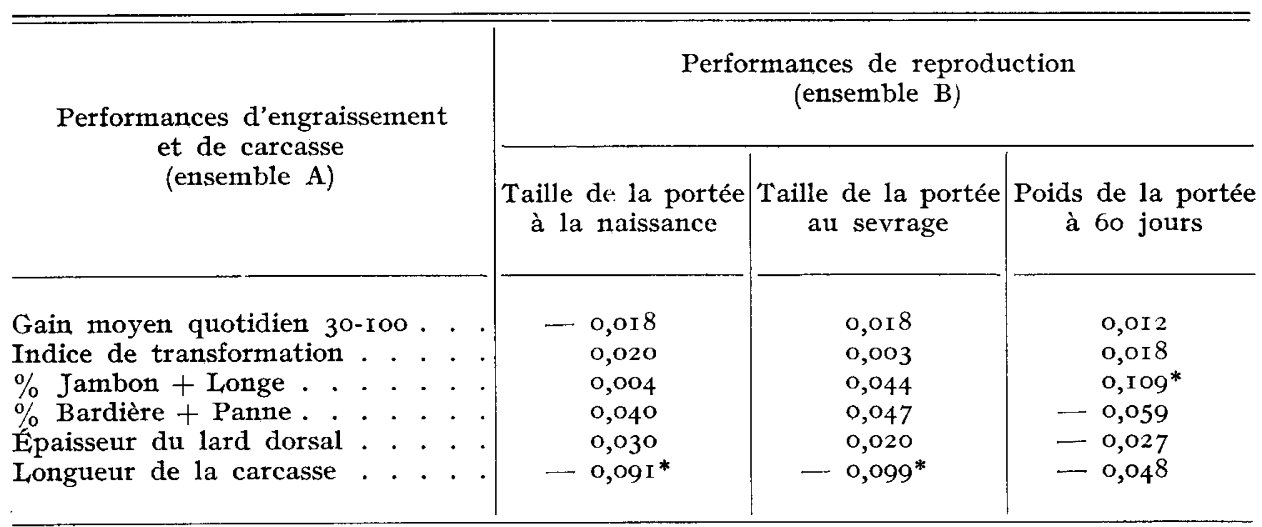

* Corrélation significative $(\mathrm{P}<0,05)$.

Trois à cinq fois plus élevées que les précédentes, les corrélations génétiques (tabl. 3) accentuent ces tendances sans qu'il soit possible de leur attribuer une valeur absolue en l'absence de l'estimation rigoureuse de leur intervalle de confiance. Toutefois, en supposant fixées les valeurs des héritabilités, on peut admettre que les corrélations génétiques obtenues en multipliant RAB par une constante, obéissent approximativement aux mêmes lois de signification que les corrélations du tableau 2 .

TABLEAU 3

Corrélations génétiques entre performances d'engraissement et de carcasse $(A)$ et performances de reproduction $(B)$

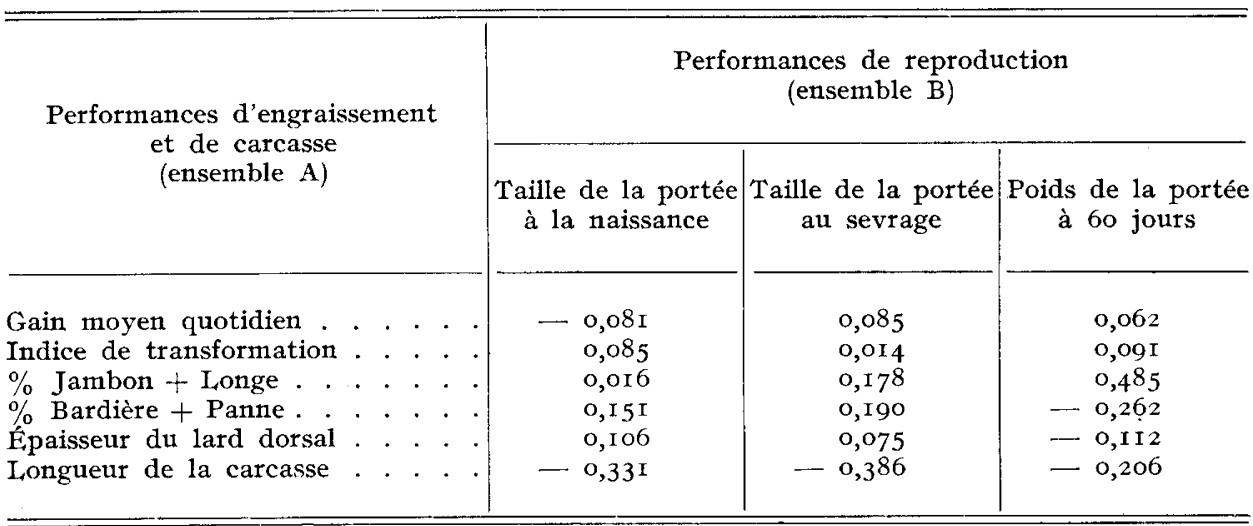


Très faibles, les corrélations canoniques (tabl. 4) montrent que la reproduction est pratiquement indépendante de la croissance $\left(\rho^{2} \mathrm{~A}_{1} \mathrm{~B}=0,002\right)$ et de la composition corporelle $\left(p^{2} A_{2} B=0,028\right)$. Les coefficients des combinaisons linéaires liées par ces corrélations figurent également au tableau 4 .

TABLEAU 4

Premiers coefficients de corvélation canonique (o) et coefficients des combinaisons linéaires liées par ces relations (*)

\begin{tabular}{|c|c|c|c|}
\hline $\begin{array}{l}\text { Groupe de } \\
\text { variables }\end{array}$ & Variables & $\rho_{\mathrm{A}, \mathrm{B}}^{2}=0,002$ & $\rho_{\mathrm{A}, \mathrm{B}}^{2}=0,028$ \\
\hline$A_{1}$ & 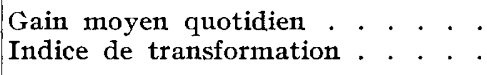 & $\begin{array}{l}2,630 \\
1,879\end{array}$ & \\
\hline $\mathrm{A}_{2}$ & 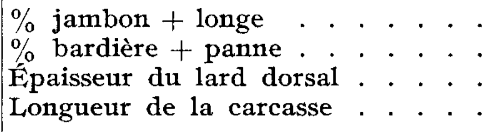 & & $\begin{array}{r}-0,224 \\
-0,376 \\
-0,611 \\
1,000\end{array}$ \\
\hline $\mathrm{B}$ & $\begin{array}{l}\text { Taille de la portée à la naissance } \\
\text { Taille de la portée au sevrage . } \\
\text { Poids de la portée à } 60 \text { jours. }\end{array}$ & $\begin{aligned}- & \mathbf{1}, 356 \\
& \mathbf{1}, 235 \\
& \mathbf{1}, 00\end{aligned}$ & $\begin{array}{r}0,023 \\
1,029 \\
-\quad 0,532\end{array}$ \\
\hline
\end{tabular}

$\left.{ }^{*}\right)$ Ces coefficients sont applicables aux variables centrées réduites.

\section{IV. - DISCUSSION ET CONCLUSION}

En s'appuyant sur des hypothèses différentes, LERNER (I954) et ROBERTSON (I956) ont développé une théorie selon laquelle la sélection naturelle favorise les phénotypes intermédiaires et toute sélection artificielle en faveur des extrêmes pour des caractères apparemment indépendants de la reproduction se traduit à long terme par la diminution de la fécondité. Des expériences de sélection réalisées sur la longueur du thorax, des ailes ou le nombre de soies abdominales de Drosophila Melanogaster (ROBERTSON et REEVE, I952; CLAYTON et ROBERTSON, I957) ont permis de vérifier cette théorie. Des expériences analogues réalisées chez la volaille par LERNER et DEMPSTER (I957) sur la longueur du tarse de la poule, par Nordskog et FESTING (I962), SiEGEL (I963) et MERRIT'T (I968) sur le poids corporel de la poule ou de la dinde (OGASAwara et al., I963) ont montré à long. terme une réduction de la ponte et du taux d'éclosion.

Cette théorie n'a pas été vérifiée aussi clairement chez les mammifères pour lesquels on ne dispose en général que d'expériences plus rares et plus brèves. Cependant, chez la souris, espèce pour laquelle "l'effet maternel " sur la taille de la portée est important (FALCONER, I963; EISEN, r970), plusieurs expériences de sélection ont montré que la taille de la portée ou le taux d'ovulation sont étroitement associés génétiquement au poids corporel ou à la vitesse de croissance (Mac ARTHUR, I949; FALCONER, I955 et I960; RAHNEFELD et al., I966; LAND, I970). 
La nature des liaisons entre la croissance ou le développement et la reproduction chez le Porc reste très discutée. Selon MoHLER (I933) et HeTzER et BRIER (I940), le développement corporel à l'intérieur d'une race favorise les performances de reproduction; par contre, après II années de sélection pour la Iéduction du poids corporel à I40 jours (création d'un porc miniature) DETTMERS et al. (I965) n'observent aucune diminution de la taille de la portée. Se basant sur plus de 4500 portées, appartenant à 38 lignées consanguines, Drckerson et al. (I954) émettent l'hypothèse d'existence d'une liaison génétique négative entre vitesse de croissance et taille de la portée. Bien qu'elle soit en accord avec les résultats de DillaRd et al. (I962), cette hypothèse est démentie par ceux de Cockerham (I952), Robison et al. (r960) et de VoGT et al. (r963) qui trouvent une corrélation génétique faible mais positive entre le poids à I $_{54}$ jours ou le gain moyen quotidien et la taille de la portée; par ailleurs, FREDEEN et PLANCK (I963) ainsi que BUCHENAUER (I970) notent l'absence de liaison entre ces carac. tères; ce dernier auteur trouve en plus des corrélations faibles mais négatives entre l'indice de consommation et la taille ou le poids de la portée. Si une idée générale doit être dégagée de ces études, elle est nettement en faveur de 1'indépendance génétique de la croissance et de la reproduction comme nous l'indiquent nos propres résultats.

Avant que ne se généralise l'emploi de méthodes de détermination objectives de la composition corporelle, des auteurs ont étudié l'influence de différentes mensurations de la truie sur ses qualités d'élevage. GRÈve (I938) et RosenHaHN (I958) n'observent pas de corrélations significatives entre ces deux groupes de caractères. Par contre HEROLD (I959) conclut que les truies les plus "sveltes" et les plus "maigres" sont les plus fertiles et les meilleures laitières. En se basant sur des données plus objectives, FREDEEN et Plank (I963) et Schal BERT (I965) observent une corrélation négative entre l'épaisseur du lard dorsal et la taille de la portée; toutefois, les premiers auteurs montrent que la taille de la portée est indépendante de la longueur de la carcasse et de la surface de la noix de côtelette. Buchenauer (I970), de son côté, ne trouve aucune corrélation significative entre la taille ou le poids de la portée d'une part, la longueur ou l'épaisseur du lard d'autre part. Après 5 générations de sélection sur la longueur de la carcasse, DuckWORTH et HoLmes (Ig68) n'observent aucune variation de la taille de la portée. Ces résultats semblent montrer l'indépendance de la longueur et de la taille de la portée alors que les nôtres laissent apparaître des corrélations négatives entre ces deux caractères; le sens de ces corrélations demande donc à être confirmé par d'autres études. Au terme de 5 générations de sélection contre l'épaisseur du lard dorsal, GRAY et al. (r965) sur la race Poland China et BERruecos et al. (I970) sur une lignée synthétique aboutissent à des conclusions différentes. Alors que les premiers auteurs notent l'indépendance de l'adiposité et des performances de reproduction, les seconds observent une diminution significative de la taille de la portée. Une corrélation légèrement positive $(r=0,09)$ avait d'ailleurs été établie entre adiposité et taille de la portée par DILLARD et al. (Ig62) à l'occasion d'une expérience de sélection sur le rapport épaisseur du lard/poids vif. Enfin, Heitzer et Miller (I970) montrent qu'après I2 générations de sélection "montante " et " descendante " sur l'épaisseur du lard dorsal en race Yorkshire et Duroc, 
les tailles des portées à la naissance et au sevrage ne diffèrent pas significativennent de celles qui sont observées dans les lignées témoins. Ces résultats semblent indiquer que la sélection contre 1'adiposité ne peut diminuer que très légèrement la fécondité des truies.

Tous ces résultats restent fragmentaires et parfois contradictoires. Ils permettent cependant de conclure que chez le Porc, les caractères de production sont génétiquement indépendants ou très faiblement associés aux performances de reproduction. Par conséquent, l'orientation actuelle de la sélection ne devrait pas, du moins à court terme, être préjudiciable à la valeur reproductive des truies.

$$
\text { Reçu pour publication en novembre } 1970 .
$$

\title{
REMERCIEMENTS
}

L'auteur tient à remercier MM. J. Lefebvre P. Mérat, et L. Ollivier de leurs conseils et. suggestions.

\author{
SUMMARY
}

\section{REI,ATIONSHIP BETWEEN FATTENING AND CARCASS PERFORMANCES}

\author{
AND LITTER PERFORMANCES IN PIGS
}

Litter performances of 1140 Large White sows and fattening and carcass characteristics of 3 of their offspring (full-sibs) raised in progeny testing stations have been studied. Intra-sire and station correlations are low and generally not significant. However, three of these correlations are exceptions $(\mathrm{P}<0.05)$ as follows: relationship between body length and litter size at birth $(r=-0.09)$ and weaning $(r=-0.10)$, and between lean-cut $\%$ and litter weight at 60 days $(r=0.11)$. Even though more important, genetic correlations have the same significance as the previous ones. "First canonic correlation coefficients" show that reproduction is practically independent from growth $\left(\rho_{1}^{2}=0.002\right)$ and from carcass characteristics $\left(\rho_{1}^{2}=0.028\right)$.

In conclusion, these results, as well as those in the literature, lead us to consider litter performance as independent, or very slightly correlated to " production traits ". Consequently, the intensive selection presently applied to growth and body composition, at least on a short term, should not depress the reproductive value of sows.

\section{RÉFÉRENCES BIBLIOGRAPHIQUES}

Berruecos J.M., Dillard E.U., Robison O.W., I97o. Selection for low backfat thickness in swiné. J. Anim. Sci., 30, $844-848$.

BUCHENAUER D., I970. Untersuchungen über die Aufzuchtleistungen eigenleistungsgeprüfter und ungeprüfter Sauen, sowie die Mastleistung und den Schlachtkörperwert ihrer Nachkommen. Giessener SchrReihe Tierz. Haustiergenet., 28, x-99.

Clayton G.A., RoBerTSON A., I957. An experimental check on quantitative genetical theory. IIThe long term effects of selection. J. Genet., 55, I52-170.

Clayton G.A., Knight G.R., Morris J.A., Robertson A., I957. An experimental check on quantitative genetical theory. III- Correlated responses. J. Genet., 55, I7I-I8o.

Cockerham C.C., 1952. Genetic covariance among characteristics of swine. J. Anim. Sci., 11, $73^{8}$. (Abstr.).

Dettmers A.E., Rempel W.E., Comstock R.E., 1965. Selection for small size in swine. J. Anim. Sci., 24, $216-220$.

Dickerson G.E., Blunn C.T., Chapman A.B., Kottmann R.N., Krider J.L., Warwick E. J., Whatley. J.A. (Jr), I954. Evaluation of selection in developing inbred lines of swine. Res. Bull. Mo. agric. Exp. Sta., (55I). 
Dillard E.U., Robison O.W., LEGates J.E., I962. Selection for low backfat weight ratio in swine. J. Anim. Sci., 21, 97 I (Abstr.).

Duckworth J.E., Holmes W., 1968. Selection for carcass length in latge White pigs. Anim. Prod., 10, $359 \cdot 372$.

Fisen E.J., I970. Maternal effects on litter size in mice. Can J. Genet. Cytol., 12, $209-2 \mathrm{r} 6$.

FALCONER D.S., 1955. Patterns of response in selection experiments with mice. Cold spring harbor. Symp. Quant. Biol., 20, 178-196.

Falconer D.S., 1960. The genetics of litter size in mice. J. Cell. Comp. Physiol., 56 (suppl. I), I 53-I67.

FALCONER D.S., I963. Maternal effects and selection response. XI Intern. Congr. Genet., 3, 763-774.

Fredeen H.T., Planck R.N., 1963. Litter size and post-weaning performance in swine. Canad. $\int$. Anim. Sci., 43, I35-142.

Gray R.C., Tribble L.F., Day B.N., LASley J.F., 1965. Five generations of selection for thinner backfat. J. Anim. Sci., 24, 848 (Abstr.).

GREve H., 1938. Untersuchungen über die Korrelationen zwischen den Körperbau den Produktionseigenschaften und den Leistungen des Hoyaerer Schweines. Thèse, Hanovre.

HeROLD I., 1959. Evaluation of types of conformation of Hungarian White meat pigs in selection. Debrecen, merogazdas. Akad. Tudoman Evk., 285-308.

Hetzer H.O., Brier G.W., I940. Extent to which type differences among swine affect litter size. Am. Soc. Anim. Prod., I939-1940, r35-138.

Hetzer H.O., Miller P.H., 1970. Influence of selection for high and low fatness on reproductive performance of swine. J. Anim. Sci, 30, $48 \mathrm{I}-495$.

Kendall M.G., 1957. A course in multivariate analysis, Griffin, London.

I.AND R.B., 1970. Genetic and phenotypic relationships between ovulation rate and body weight in the mouse. Genet. Res., 15, I7 I-182.

L,EGAULT C., I970. Étude statistique et génétique des performances d'élevage des truies de la race Large White. II. - Effet direct du verrat, héritabilité, corrélations. Ann. Génét. Sél. anim., 2, 209-227.

LERNER I.M., I954. Genetic homeostasis, 134 p., Oliver and Boyd, Edinburgh.

I,EKNER I.M., DEMPSTER E.R., I95I. Attenuation of genetic progress under continuous selection in poultry. Heredity, 5, 75-94.

L,USH J.I., 1945. Animal brecding plans. Iowa state college Press, Ames.

M ac Arthur J. W., I949. Select.on for Small and large body size in the house mouse. Genetics 34, I 54, 209 .

MERI IT E.S., I968. Genetic alteration of growth pattern and correlated responses in the fowl. XII Int. Congress of Genetics., 1, 273.

Mohler J.R., 1933. Report of the chief of the Bureau of animal industry, 1933 U.S. Dept. Agric. Bureau Anim. Ind., 47 p.

Nordskog W., Festing M., I962. Selection and correlated responses in the fowl. XII World's Poultry Congress, 25-29.

Cgasawara F.X., Abplanalp H., Asmundson V.S., 1963. Eiffect of selection for body weight on reproduction in Turkey hens. Poult. Sci., 42, 838-842.

Cllrvier L., I969. Paramètres génétiques du porc Latge White Français. Journées de la Recherche porcine en France, Paris 20-21 février, 7-12.

Rahnefeld G.W., Comstock R.E., Madhosingh Napuket S.R., r966. Genetic correlation between growth rate and litter size in mice. Genetics, 54, I423-1429.

RoBERTSON F.W., r956. The effect of selection against extreme deviants based on deviation or on homozygosis. J. Genet., 54, 236-248.

Robertson F.W., and ReEve E.C.R., 1952. Studies in quantitative inheritance. I- The effects of selection of wing and thorax lenght in Drosophila melanogaster. J. Genet., 50, 4 I 4448.

Robison O.W., Chapman A.B., Self H.I., 1960. Swine selection indexes including live animal measurements as indicators of carcass merit. J. Anim. Sci., 19, ro24-rozo.

RosenhaHN W., I958. Untersuchungen über Zuzammenhänge Zwischen Körperformen und Züchtleistungen bei Schweinen. Zuchtungskunde, 30, I55-164.

SCHALbert J., 1965. Backfat thickness and the amount of fat in progeny of Mangalitsa sows differing in fecundity. Rebrecen. agrartud. Foisk. Tudoman Közl., 11, 107-114.

Sieger P.B., I963. Selection for body weight at 8 weeks of age. 2-Correlated responses of feathering, body weight and reproductive characters. Poult. Sci., 42, 896-905.

Vogt D.W., Comstock R.E., REMPeL W.E., r963. Genetic correlation between some economically important traits in swine. J. Anim. Sci., 22, $2 \times 4-217$. 